\title{
Trapped mode resonances in metalo-dielectric structures with electric asymmetry materials
}

\author{
MiHAi D. RotARU, JAN K. SYKULSKI \\ School of Electronics and Computer Science \\ University of Southampton, Southampton, SO17 1BJ, UK \\ e-mail:mr@ecs.soton.ac.uk,jks@soton.ac.uk
}

(Received: 02.07.2010, revised: 02.12.2010)

\begin{abstract}
This paper investigates the possibility of exciting high quality trapped resonant modes on frequency selective surfaces consisting of identical sub-wavelength metallic inclusions (symmetrically split rings) with no structural asymmetry but exhibitting electrical asymmetry. The electrical symmetry is broken by using different dielectric substrates. The existence of such anti-symmetric trapped mode on geometrical symmetric structure is demonstrated through numerical simulation. Numerical results suggest that the high quality factor observed for these resonant modes is achieved via weak coupling between the "trapped modes" and free space which enables the excitation of these modes.

Key words: trapped mode resonance, high quality factor, electromagnetic metamaterials, electrical asymmetry
\end{abstract}

\section{Introduction}

Electromagnetic metamaterials and their potential applications have attracted significant attention from the scientific community in recent years. The research is driven by the opportunity to achieve electromagnetic properties that lead to new phenomena such as negative refraction, perfect magnetic surface or cloaking [1], which have no equivalents in natural world.

Most of such 'exotic' electromagnetic response of metamaterials is due to their resonant behaviour; hence achieving a high-quality factor resonance is essential to obtain an efficient metamaterial based device. Typically, a metamaterial cell comprises a metallic structure - in which currents can be induced - and a supporting dielectric substrate. Depending on the induced current distribution in the conducting region, different resonance modes can be excited within these cells. Most of these resonant structures do not have a very high resonant quality factor because they suffer significant radiation losses. Moreover, due to their relative small size with respect to the wavelength of operation, the metamaterial cell structures are not

\footnotetext{
${ }^{*}$ This is extended version of a paper which was presented at the 21st Symposium on Electromagnetic Phenomena in Nonlinear Circuits, Essen-Dortmund, 29.06-02.07, 2010.
} 
able to provide a large enough volume for the confinement of electromagnetic field necessary to support high quality factor resonances. However, most recent theoretical analysis and experimental results have shown that high quality factor resonances involving trapped modes are possible in metamaterials, providing a certain small structural (geometrical) asymmetry is introduced to the shape of their structural conducting elements [2-5].

In this work it is shown through numerical simulation that similar effects can be achieved without breaking the geometrical symmetry of the resonator but instead by introducing an electrical asymmetry through a combination of substrates with different dielectric properties.

\section{Frequency selective surface}

\subsection{Frequency selective surface layout}

The structures studied in this work are based on a split ring configuration arranged in a periodic array as shown in Figure 1. The split rings are assumed to be made of copper, with a radius of $6 \mathrm{~mm}, 0.8 \mathrm{~mm}$ width and $35 \mu \mathrm{m}$ thickness. The substrate thickness is $1.5 \mathrm{~mm}$ and cell dimensions are $15 \times 15 \mathrm{~mm}$. The substrate is assumed to have a complex dielectric permittivity $\varepsilon=4.07+j 0.05$. The ring is split along the $y$ direction. By removing the conducting material within the two $20^{\circ}$ angles as illustrated in Figure 1, two equal conducting arcs are obtained. The array is considered to be infinitely long in the $x$ and $y$ directions. Such an arrangement will not normally diffract a normal incident electromagnetic wave for frequencies below $15 \mathrm{GHz}$. If the normal incident wave is polarised along the $y$ direction it is expected that the frequency selective surface with the shape, sizes and properties described above will reflect the incident wave at a frequency at which the wavelength is equal to the circumference of the ring. A wave polarised in the $x$ direction will be reflected in the same way but at a higher frequency when the wavelength is equal to the length of the arcs.
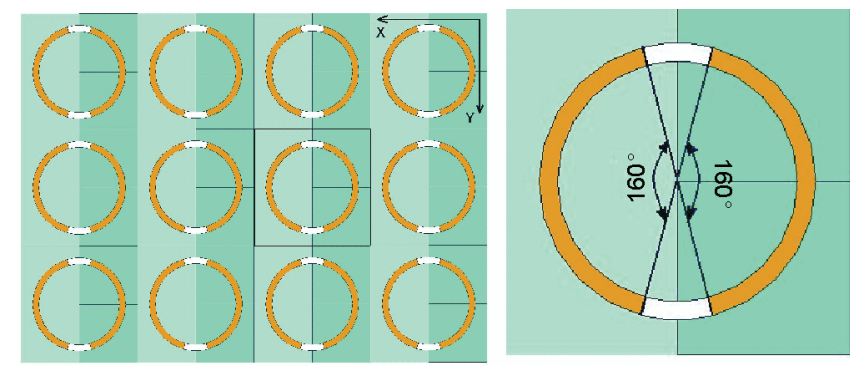

Fig. 1. Planar array of split ring resonators and elemental cell

\subsection{Numerical model set-up}

To confirm the behaviour a 'full wave' simulation using commercially available software (CST Microwave Studio) was undertaken. A model of the frequency selective surface described in previous sub-section was built in the 3D modeller. This structure can be simulated by imposing periodic boundary conditions and assuming an infinite periodic array of elemen- 
tary cells. The array from Figure 1 was illuminated with a transverse electromagnetic wave for two polarizations, in $x$ and $y$ directions respectively. The transverse wave was introduced into the model using Floquet ports. In this work only the fundamental Floquet modes $\mathrm{TE}(0,0)$ and $\operatorname{TM}(0,0)$, which are linear polarised waves, were used. The $\operatorname{TE}(0,0)$ Floquet mode is polarised in $y$ direction while $\operatorname{TM}(0,0)$ is polarised along $x$ direction. The problem was solved in frequency domain using a tetrahedral mesh. A frequency sweep between 3 and $15 \mathrm{GHz}$ was considered and the transmission and reflection through and from the split rings array were calculated.

\subsection{Numerical results}

The numerical results confirmed the expected behaviour, mainly that no diffraction is observed in this band. Figure 2 presents the transmission and reflection responses for the two polarizations considered.

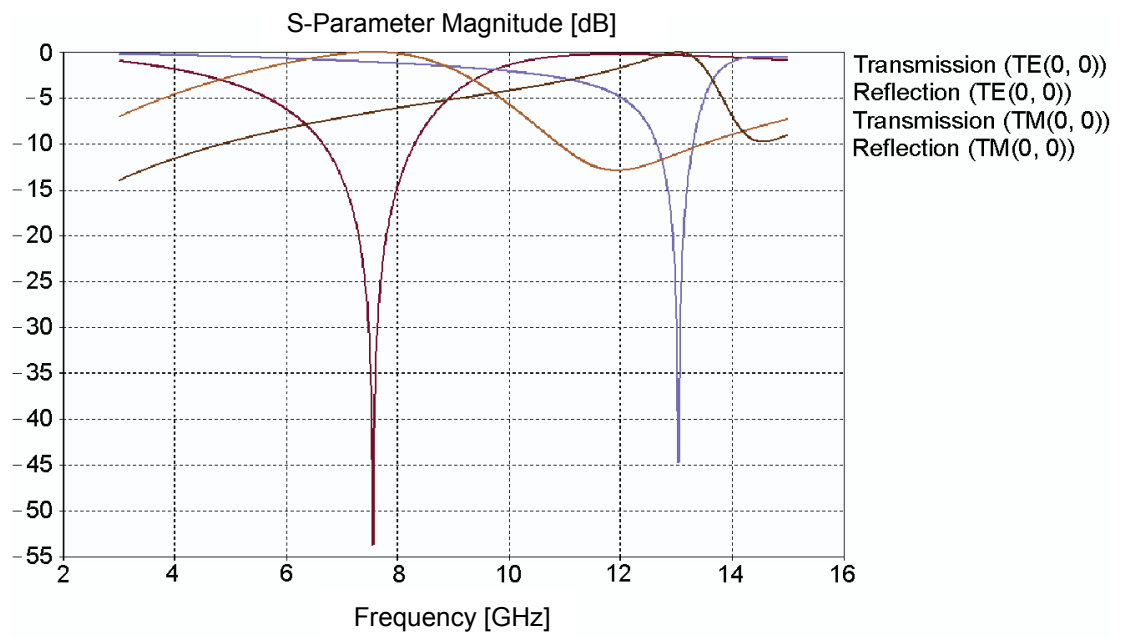

Fig. 2. Transmission and reflection response for the $\operatorname{TE}(0,0)$ and $\operatorname{TM}(0,0)$ fundamental Floquet modes

The resonances observed at $7.5 \mathrm{GHz}$ and $13 \mathrm{GHz}$ are due to the interaction of two counter propagating waves. For example, at $7.5 \mathrm{GHz}$, the wavelength of the incoming $\mathrm{TE}(0,0)$ polarised wave is equal to the circumference of the ring; hence at this frequency a dipole-like resonance will be excited on to the arcs. Figure 3 a illustrates the current distribution on the surface of the two arcs at this frequency. It can be noticed that the currents on the two sides are equal and in phase. The structure behaves in this regime like a small radiating dipole. The wave that is radiated by the arcs will be in anti-phase with the incoming wave. This is observed as a strong reflection from the frequency selective surface. A similar effect exists at $13 \mathrm{GHz}$ but for the $\mathrm{TM}(0,0)$ polarization. Both of these resonances can be considered as an interference of counter propagating waves in the same scattering channel due to the superposition principle, and are known as Breit-Wigner resonances. 

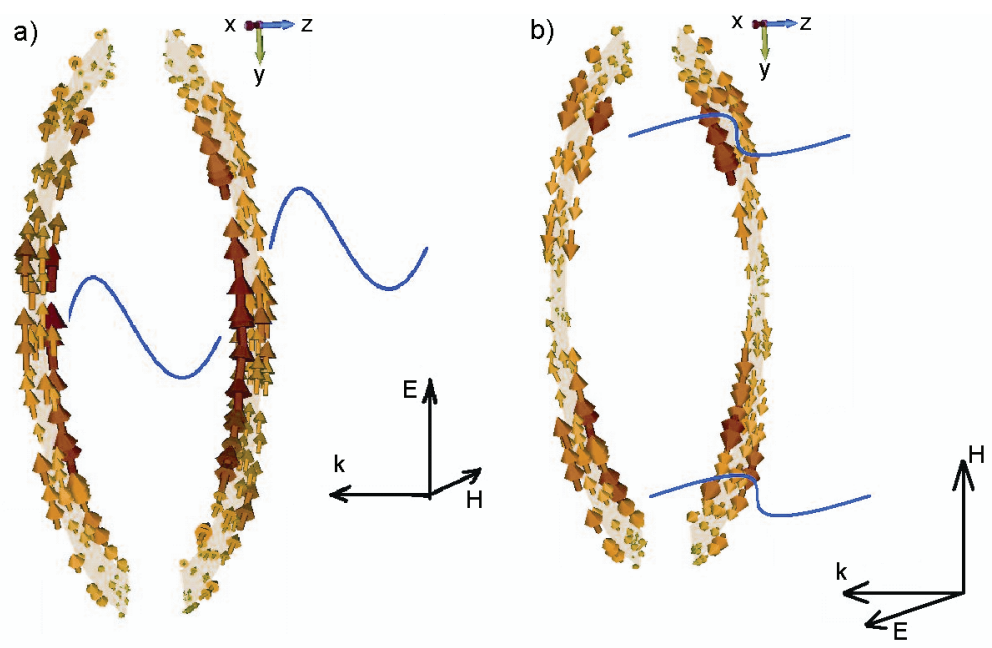

Fig. 3. Current distribution on the metallic arcs: a) $\mathrm{TE}(0,0)$ polarisation; b) $\operatorname{TM}(0,0)$ polarisation

The quality factor $(Q)$ of the resonance can be defined as the ratio of the resonant frequency and the width of the resonance measured at $3 \mathrm{~dB}$ below the maximum (1).

$$
Q=\frac{f_{\text {resonance }}}{B W_{3 d B}} .
$$

Using this definition $Q$ may be calculated for the two resonances: $Q_{\mathrm{TE}(0,0)}=1.66$ and $Q_{\mathrm{TM}(0,0)}=5$. These two values are relatively low but typical for such structures that are strongly coupled to the free space. To increase the quality factor, and hence the efficiency of the metamaterials, the radiation losses have to be reduced

\section{Trapped mode resonances}

\subsection{Electric asymmetric resonator}

The behaviour described in the previous section can be changed by breaking the geometrical symmetry of the metallization. Asymmetrically split rings have been found to diffract normal incident waves at relatively low frequencies [2], where the authors have shown the existence of unusually strong quality resonant modes named "trapped modes" on these geometrical asymmetric structures. In their work the authors have fabricated and simulated a frequency selective surface with a structural asymmetric split ring resonator. The asymmetry was introduced by having the two arcs of slightly different lengths. The response of such a structure has been confirmed by our numerical simulation. Analyzing these numerical results in more detail revealed that the existence of these extremely narrow transmission and reflection pass and stop bands are due to the electrical asymmetry of the system rather than the geo- 
metrical one. Therefore it should be possible to create a geometrically symmetric structure but with electrical asymmetry that supports similar trapped mode resonances.

A 3D model was developed to show the existence of the trapped modes on such geometrically symmetrical cells. In our numerical experiment we have kept the split rings symmetrical but changed the substrate in such a way that half of it has a different value of the dielectric constant to the other half (Figure 1). Taking into account the two wave polarizations described above, two possibilities have been considered of how the substrate could be partitioned. Our simulations, however, have shown that only one situation depicted in Figure 1 creates a response similar to the asymmetric split rings arrangement. Considering a case for which half of the substrate has a complex dielectric permittivity $\varepsilon=4.07+j 0.05$ and the other half $\varepsilon=5+j 0.05$, for an electromagnetic wave incident to the array with a polarization normal to the mirror line ( $y$ polarization in Figure $1-\mathrm{TE}(0,0)$ Floquet mode), the reflection and transmission responses create a very different situation than the symmetric split rings with homogenous substrate (Figure 4). The graph of the reflection response for the new electrically asymmetric case reveals a very sharp resonance just bellow $6 \mathrm{GHz}$ (Figure 4). Two weaker resonances, corresponding to peaks in the reflection response, are also present at 5.8 and $7.3 \mathrm{GHz}$, respectively. The transmission plot shows a very narrow peak reaching $-4.78 \mathrm{~dB}$. Its width is only $0.36 \mathrm{GHz}$ as measured at $3 \mathrm{~dB}$ below the maximum. The quality factor for this sharp resonance was calculated using (1) at the centre frequency of $5.85 \mathrm{GHz}$. The resultant value of 16 is larger by one order of magnitude as compared with the $Q_{\operatorname{TE}(0,0)}=1.6$ calculated in the previous section for the Breit-Wigner resonance which is typical for most metamaterials based on lossy PCB substrates.
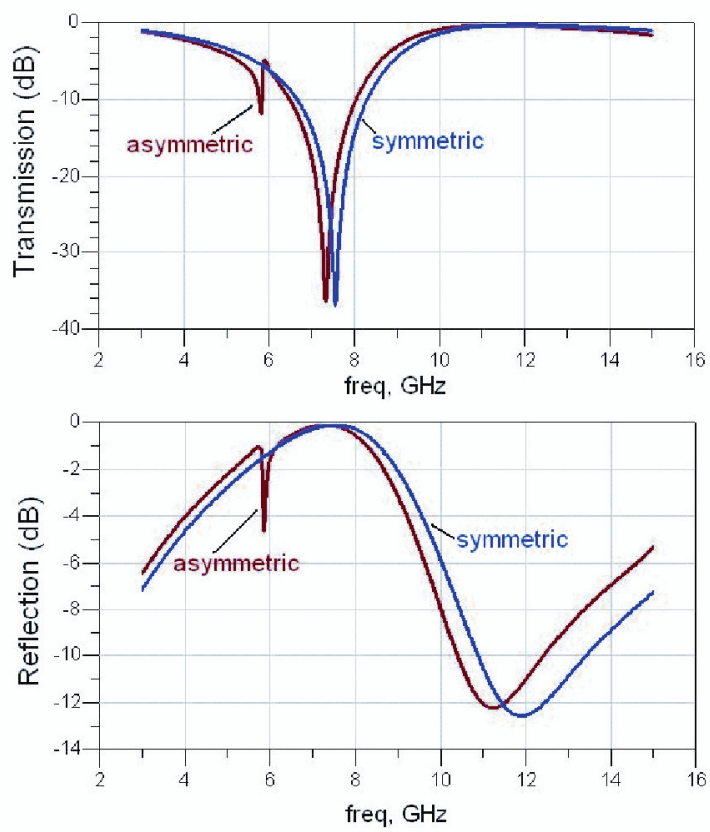

Fig. 4. Comparison of calculated transmission and reflection responses of electrically symmetric and asymmetric structures for a $\operatorname{TE}(0,0)$ fundamental Floquet mode 
To understand the resonant nature of the response of the electrically asymmetric structure several more simulations were undertaken. Different values for the complex dielectric permittivity were considered. It was decided to keep half of the substrate fixed with $\varepsilon=4.07+j 0.05$ and increase the real part of the complex permittivity of the other half. The results in terms of the transmission and reflection are shown in Figure 5. It is clear that by increasing the difference between the real parts of the permittivity of the two halves the sharp resonance is shifting towards lower frequency and the $Q$ factor decreases.
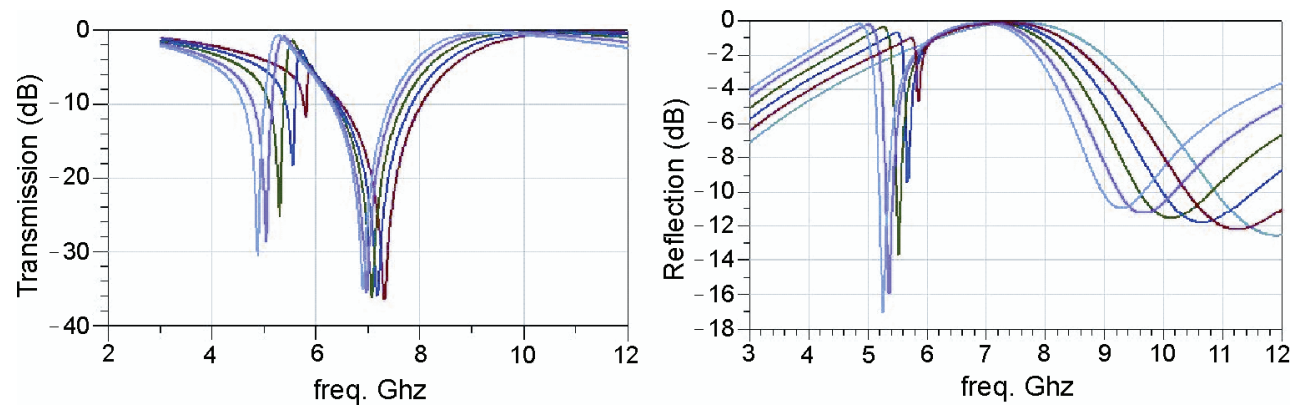

Fig. 5. Change in the transmission and reflection response due to the increase difference between real parts of permittivity

The current distribution within the cell has also been be analysed. The reflection and transmission response of the frequency selective surface for the $\operatorname{TE}(0,0)$ mode, when $\varepsilon=4.07+j 0.05$ in one half and $\varepsilon=9.4+j 0.002$ in the other half of the cell, is presented in Fig. 6.

The current distributions corresponding to the resonant modes are also shown. For the first resonant mode, when the frequency selective surface reflects the incoming wave, the simulation results show that the two arcs have currents that are anti-phase but one of the currents dominates. The current dominating the response is excited on the arc that is on the half of the ring with lower permittivity.

For the second resonance, the two arcs support currents that are almost equal in amplitude but in anti-phase. The scattered electromagnetic field produced by such a current arrangement is very weak, reducing the coupling to the free space and therefore reducing the radiation losses. The electric and magnetic fields produced by the two oscillating currents practically cancel each other out and as a result the incoming wave passes unimpeded through the surface, as clearly shown by the transmission response.

This results in very high induced currents (Figure 7) on the metallic arcs which ensure a high quality factor response. For the example shown here (Figure 7) the computed current amplitude for the second resonance is about three times larger than for the third resonance. Finally, for the third resonance, the two currents are in phase but now the current on the arc sitting on the half with the lower permittivity dominates the response. Once more the surface reflects the incoming wave. 

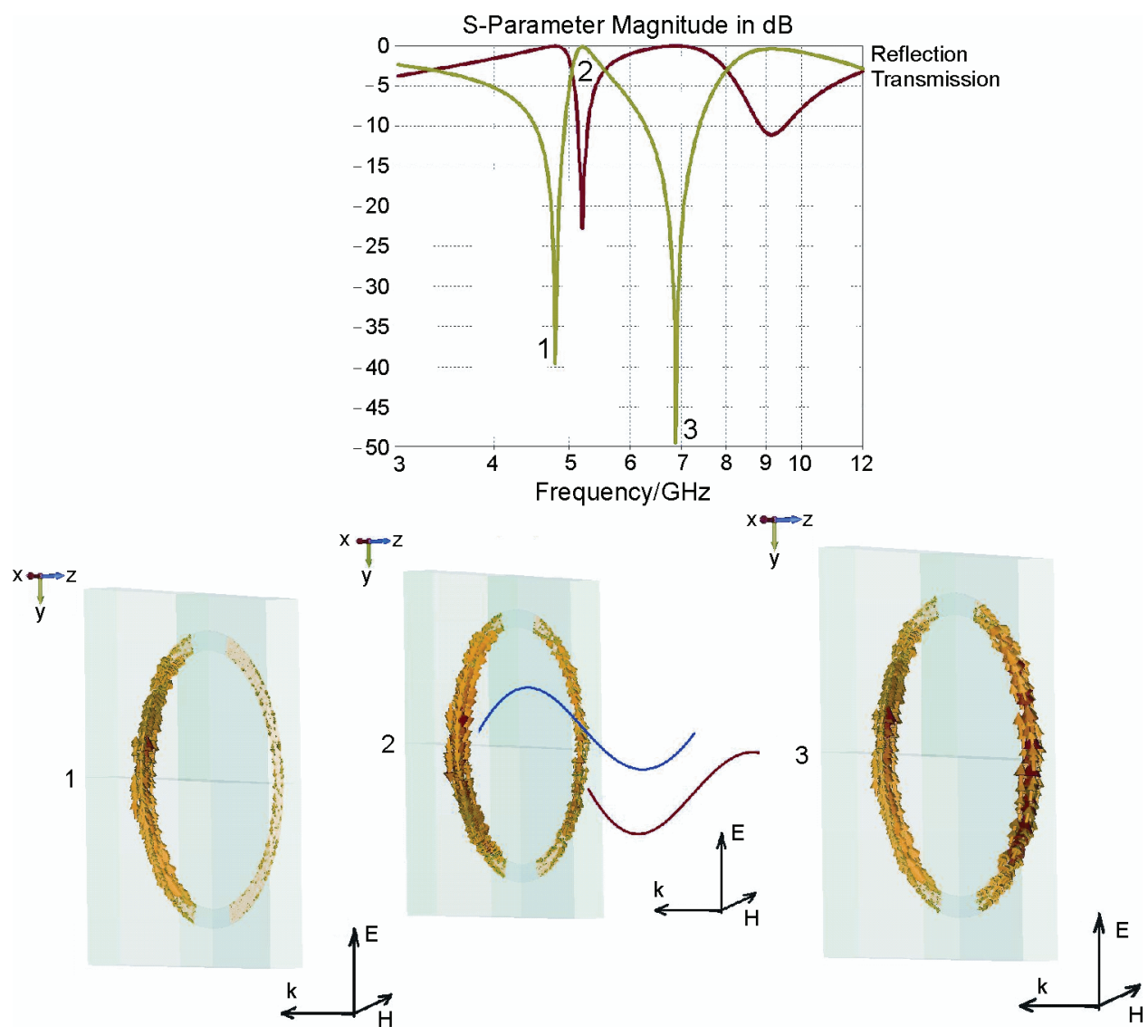

Fig. 6. Change in the transmission and reflection response due to the increase difference between real parts of permittivity
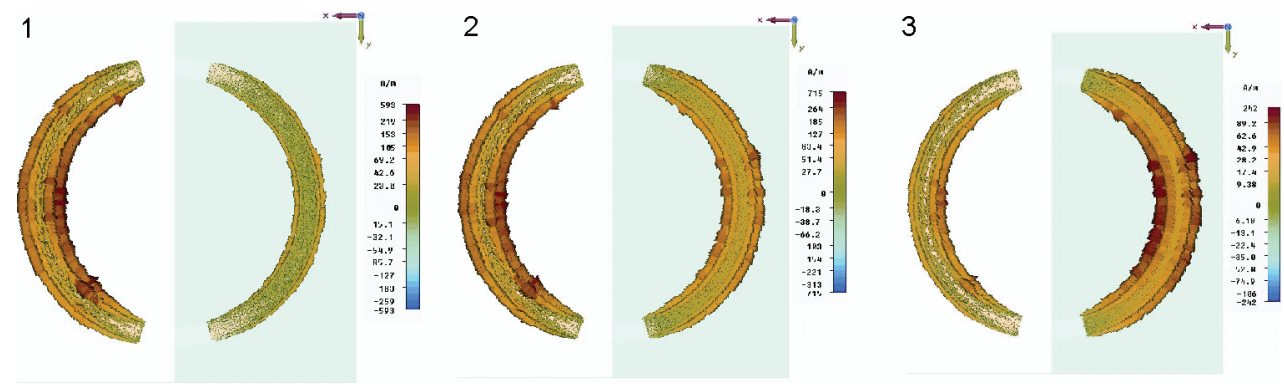

Fig. 7. Current distribution and amplitude for the three resonant modes

This type of response is not possible in an electrically symmetric structure and the high quality resonant mode excited is said to be "trapped" within the structure; hence the name "a trapped mode". These types of resonances that have close coexistence of resonant trans- 
mission and resonant reflection are part of a more general class of resonances with asymmetric profile named Fano resonances.

\subsection{Quality factor variation}

To quantify better the quality factor variation with the electrical asymmetry we have defined an asymmetry factor $\xi$ as the ratio of the values of $\operatorname{Re}(\varepsilon)$ of the substrate for the two halves of the cell $(0<\xi<1)$. The variation of the quality factor $Q$ with respect to the asymmetry factor $\xi$ is shown in Figure 8. Our numerical simulations have shown that by reducing $\xi$ the three resonant modes shift towards lower frequencies (Figure 8). We have also noted that the quality factor has a maximum of about 17 for $\xi=0.68$.

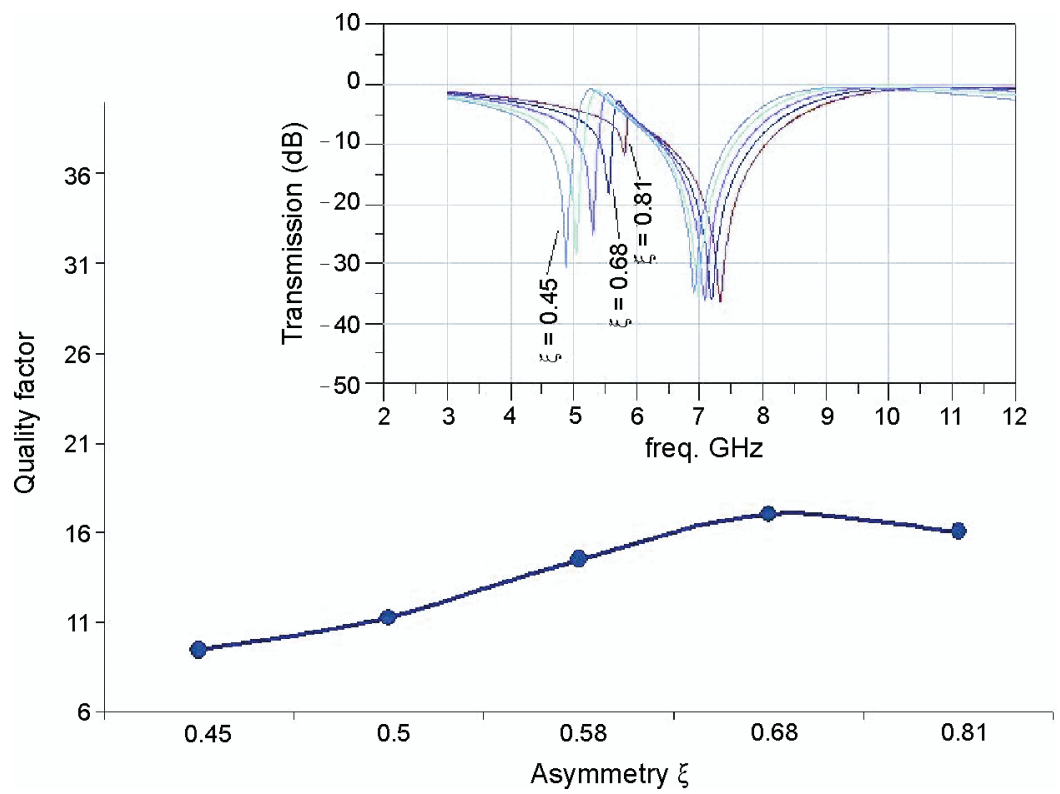

Fig. 8. Quality factor variation with asymmetry factor

\section{Conclusions}

The existence of high quality "trapped mode" resonances on geometrically symmetric structures was demonstrated through full wave simulations. The necessary condition for exciting such modes is an electrical asymmetry rather than geometrical asymmetry. The electrical asymmetry can be achieved by using structural asymmetry of the metallic 'inclusions' [2] but also by controlling the dielectric properties of the substrate on which the metallic structure are build on.

The frequency selective surfaces composed of the electrical asymmetric cells exhibit very high quality resonances and provide extremely narrow transmission and reflection pass and 
stop bands. The high quality factor achieved for this response is one order of magnitude larger than the typical values obtained for more conventional metamaterials. This is due mainly to the low radiation loss of the 'trapped mode' excited on the asymmetric structures.

Achieving trapped mode resonances will be more important for metamaterials working at terahertz frequencies where losses are significant and unavoidable. This work opens up the possibility of designing artificial structures whose electromagnetic response could be controlled and tuned trough electrical means by changing their electrical asymmetry.

\section{References}

[1] Engheta N., Ziolkowski R.W., Metamaterials, physics and engineering exploration. IEEE Press John Wiley \& Sons, Inc.: 1-37. (2006).

[2] Fedotov V.A., Rose M., Prosvirin S.L. et al., Sharp trapped-mode resonances in planar metamaterials with broken structural symmetry. Physical Review Letters 99: 147401-1-147401- 4 (2007).

[3] Khardikov V., Iarko E., Prosvirin, S. Trapped mode resonances in optical planar metamaterials with metal elements. $12^{\text {th }}$ International Conference on Mathematical Methods in Electromagnetic Theory, Odessa, Ukraine, June 29-July 02 (2009).

[4] Ourir A., Abdeddaim R., de Rosny J. (2010), Tuneable trapped mode in symmetric resonator designed for metamaterials. Progress in Electromagnetics Research, PIER-101: 115-123.

[5] Paul O., Beigang R., Rahm M. (2009), High selective terahertz bandpass filters based on trapped mode excitation. OPTICS EXPRESS 17(21): 18590-18595. 